\title{
Polymorphism of the Crystals and the Formation of Snowflakes in the Clouds
}

\author{
Yuri Pivovarenko \\ Research and Training Centre 'Physical and Chemical Materials Science' Under Kyiv Taras Shevchenko University and NAS of Ukraine, \\ Kiev, Ukraine
}

\section{Email address: \\ y.pivovarenko@gmail.com}

\section{To cite this article:}

Yuri Pivovarenko. Polymorphism of the Crystals and the Formation of Snowflakes in the Clouds. American Journal of Electromagnetics and Applications. Vol. 6, No. 1, 2018, pp. 1-5. doi: 10.11648/j.ajea.20180601.11

Received: February 18, 2018; Accepted: March 8, 2018; Published: March 26, 2018

\begin{abstract}
It was previously shown that the evaporation of the salt solution prepared in water with a positive electric potential, accompanied by the formation of cubic or rhombic crystals, and evaporation of the salt solution prepared in water with a negative electrical potential is accompanied by formation of needle-like crystals. It was also shown that the shape of salt crystals depends on the sign of the electric charge of the surface on which salt crystals are formed. It has been particularly shown that cubic or rhombic crystals formed on positively charged surfaces, while the needle-like crystals are formed on negatively charged surfaces. Knowing these dependencies is very productive, because it gives you an opportunity to establish a number of phenomena occurring in animate and inanimate nature. In order to popularize this idea, we propose the results obtained through its implementation. So, the knowledge of these dependencies allows us to explain the processes occurring in the storm glass during the weather change, as well as the formation of snowflakes in the clouds.
\end{abstract}

Keywords: Crystals, Polymorphism, Storm Glass, Snowflakes, Snowflakes Formation

\section{Introduction}

It was previously established that the shape of the crystals formed after evaporation of salt solutions depends on the sign of the electric potential of the water used for preparing such solutions. It has been particularly shown that the evaporation of salt solutions with positive electric potential is accompanied by the formation of cubic crystals (Figure 1, left) and the evaporation of salt solutions with negative electric potential is accompanied by the formation of needlelike crystals (Figure 1, right) [1, 2].

It was also shown that the shape of salt crystals depends on the sign of the electric charge of the surface on which salt crystals are formed. It has been particularly shown that cubic or rhombic crystals formed on positively charged surfaces (Figure 2, left), while the needle-like crystals are formed on negatively charged surfaces (Figure 2, right) [1].

This correlation has been very productive for the explanation of certain phenomena, the nature of which remained unclear for a long time. In particular, this correlation was previously used successfully to explain the nature of the polymorphism of crystals, underlying one of the methods of medical diagnosis [1]. Here it is shown how such correlation can be used to explain the formation of snowflakes.

It should be said that interest in the form of snowflakes and the process of their formation arose quite a long time and have survived to our time. This is confirmed by the incessant attempts to explain the physical nature of the phenomena, through which snowflakes are formed.

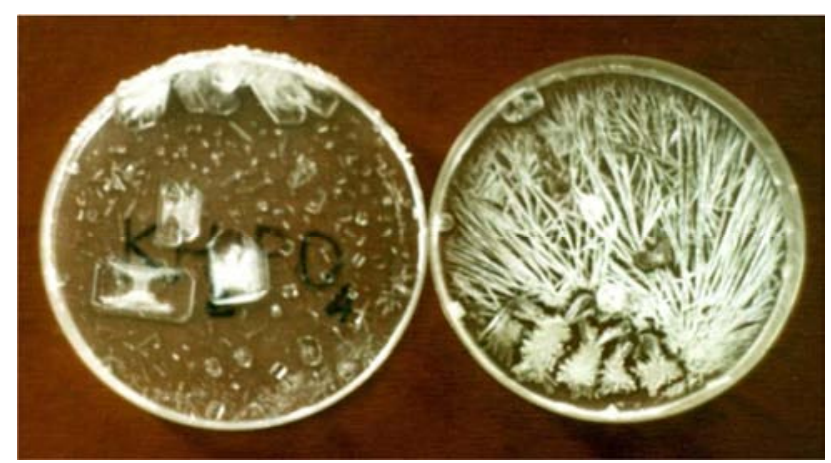

Figure 1. Left: the rhombic crystals formed upon drying of an aqueous solution of $\mathrm{KH}_{2} \mathrm{PO}_{4}$ prepared on the water with positive electric potential. Right: the needle-shaped crystals formed upon drying of an aqueous solution of $\mathrm{KH}_{2} \mathrm{PO}_{4}$ prepared on the water with negative electric potential [1, 2]. 
Despite numerous attempts to unravel the mechanism of the formation of snowflakes, it must be recognized that this mechanism remains enigmatic to this day [3-10]. So the mystery of the formation of snowflakes is a challenge for all naturalists. Therefore, the here presented results may be of interest to a wide circle of readers.

\section{Material and Methods}

The term "uncharged water" was used to designate the water, which was used as a control: it was assumed that the electric potential of this water is $0 \mathrm{mV}$. Uncharged water was obtained during storage of distilled water in a closed aluminum container, - it is considered that in such circumstances, the electric charges of water are concentrated on the outer surface of the container [11].
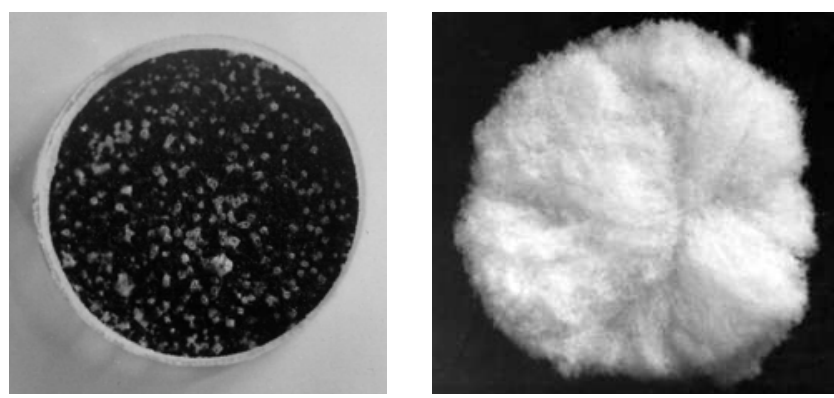

Figure 2. Left: These are the small cubic crystals formed on positively charged surface of activated carbon, pre-wetted with a solution of $\mathrm{NaCl}$. Right: These are the needle-shaped crystals formed on negatively charged surface of silica gel, pre-wetted with a solution of $\mathrm{NaCl}$; in this case, the interlacement of acicular crystals forms a kind of wool [1].

Water with a positive electric potential was obtained by filtration of uncharged water through silica gel. It is known that the silica gel exhibits the properties of a sorbent of aqueous hydroxyl ions [12].

Water with a negative electric potential was obtained by filtration of uncharged water through the activated carbon. It is known that the activated carbon exhibits the properties of a sorbent of aqueous hydrogen ions [12].

The electric potential of the electrically charged water was measured relative to the uncharged water. In fact, the electric potential of the charged water, was measured the potential flow or potential filtering [13].

\section{Results and Discussion}

\subsection{The Polymorphism of Crystals}

Using the described relationships (Figures 1, 2) [1, 2], it is possible to control the shape of the formed crystals. So, by varying the electrical potential of the water used for preparation of salt solutions and using different surfaces, it is possible to obtain crystals of different shapes. Look particularly impressive crystals that have formed after evaporation of a solution of $\mathrm{CuSO}_{4}$ (Figures 3-5).

It should be also noted that this dependence is observed not only for the salt crystals, but for some of the metals
(Figure 6). This can be seen as evidence that the shaping properties inherent in water; in turn, the shaping properties of water are determined by its electric potential.

The results presented in Figures 4-6, definitely appealing to researchers studying the phenomenon of crystallization in living matter [14]. In any case, these examples of such expressive arborization of aqueous salt solutions need to convince that the morphological characteristics of plants are determined not only by genotypes.

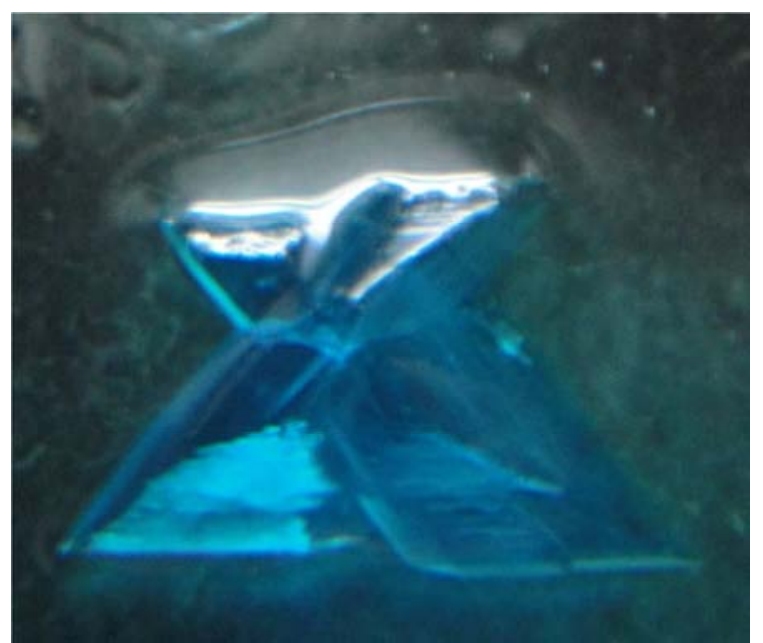

Figure 3. These are the types of crystals that may have formed on the glass surface after evaporation of a solution of $\mathrm{CuSO}_{4}$, prepared on the water with positive electric potential.
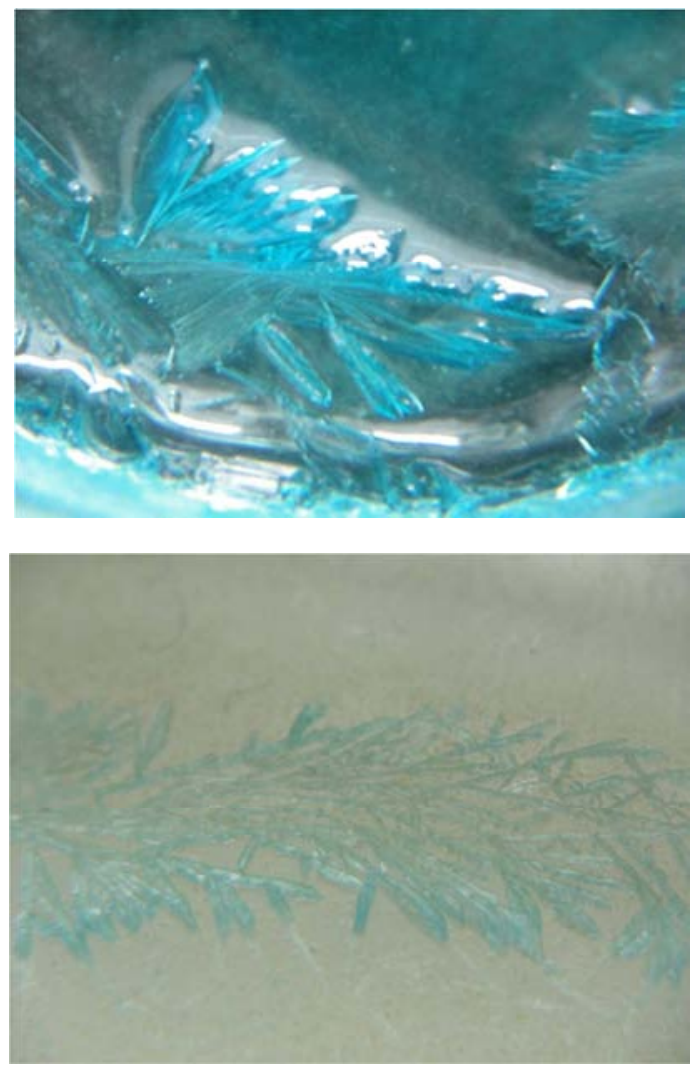

Figure 4. These are the types of plant-shaped crystals that may have formed on the glass surface after evaporation of a solution of $\mathrm{CuSO}_{4}$, prepared on the water with negative electric potential. 

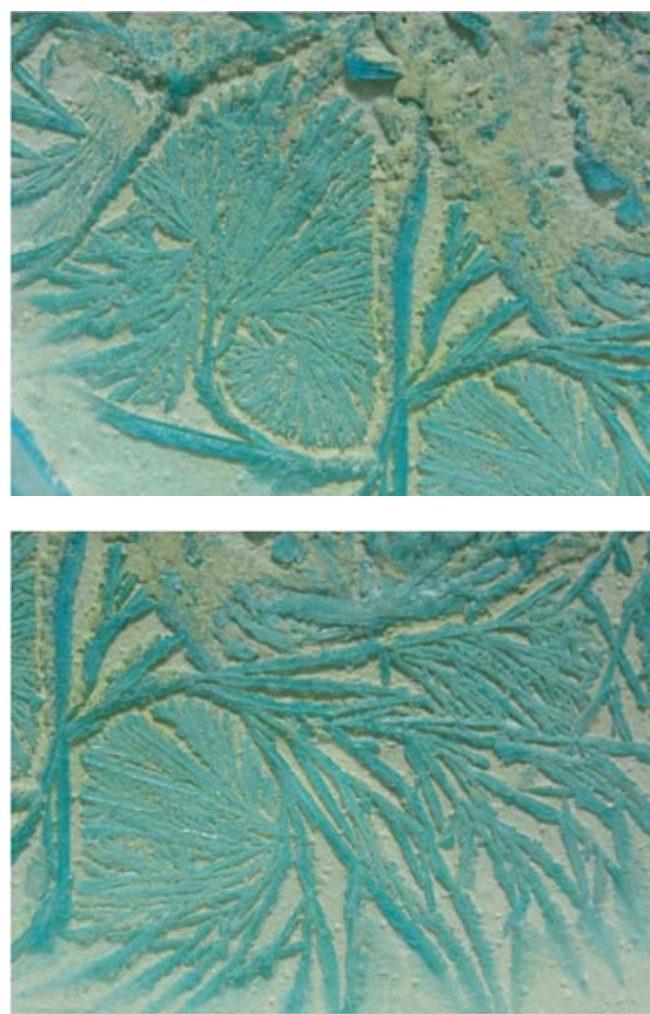

Figure 5. These are typical "iegetable"crystals formed on the surface of the paper after evaporation of a solution of $\mathrm{CuSO}_{4}$, prepared on the water with negative electric potential.

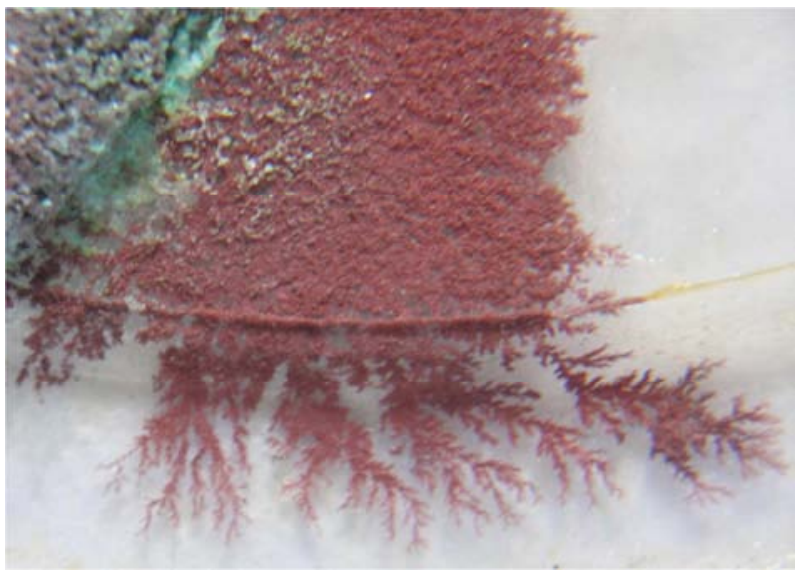

Figure 6. This is the powder of metallic copper at the boundary of aqueous solutions with positive (above) and negative (below) potentials.

\subsection{The Polymorphism of Crystals in Storm Glass}

It should be also noted that a perfect illustration of installed dependencies for many years is storm glass [15], which reflects the sign and concentration of the atmospheric charges. Especially spectacular arborization in the content of storm glass takes place at the approach of cyclones (Figure 7). It is known that cyclones are significant carriers of negative charge. Thus, direct measurements showed that the electric charges of the cyclones in Kamchatka have value: $\left(10^{3}-10^{4} \mathrm{C}\right)[16]$.

So, knowledge of the announced relationships proved to be very productive as to obtain crystals of the desired shape and to explain certain phenomena. Let's use it in the future.

\subsection{The Branching of Needle-Shaped Crystals}

The phenomenon of branching needle-shaped crystals (Figures 3-6, 8) is of particular interest both in theoretical and practical aspects.

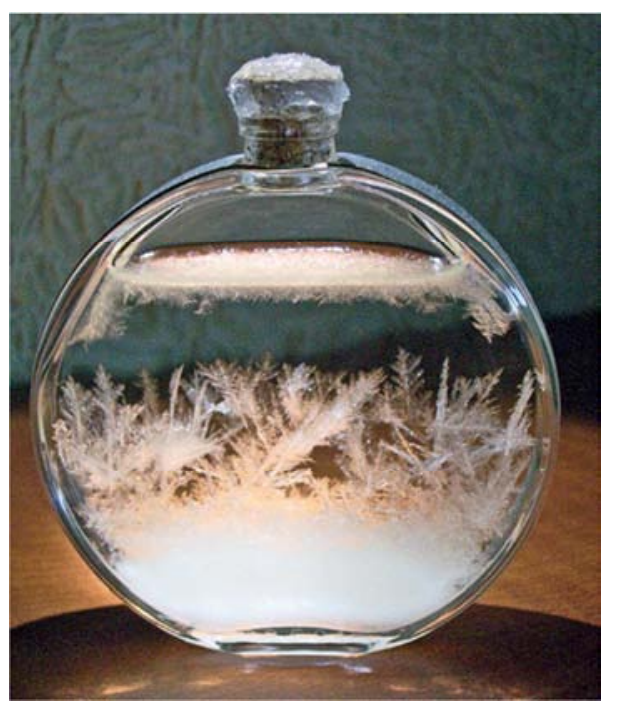

Figure 7. These are the typical tree-like crystals that form in the storm glass in front of the approaching cyclone, i.e. before the deterioration of weather.

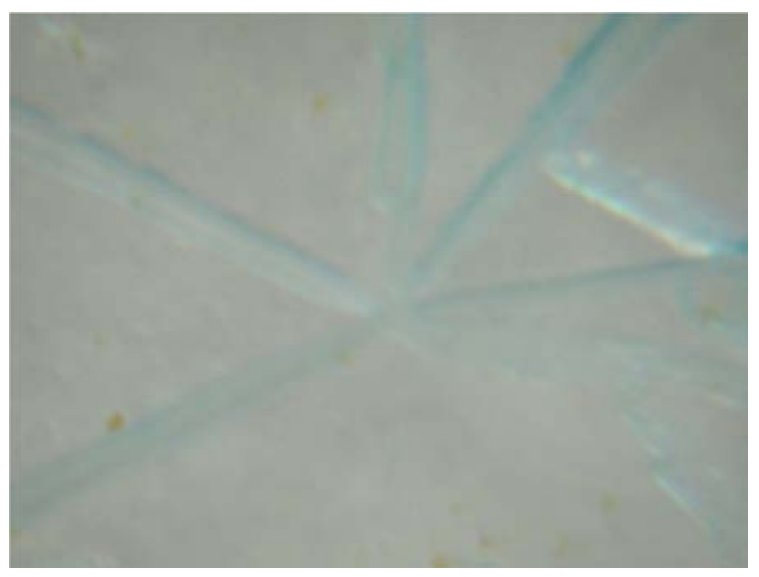

Figure 8. It is a typical branching of needle-shaped crystals.

There was an assumption that the positive charge is localized in a branch. It was also suggested that the needles rays (or needles) diverging from this point, have negative charges. The following experiment was conceived and implemented to check such assumptions.

As is known, the positive charges are moving in the direction of the light, which is defined by the Pointing vector. At the same time, the negative charges move against the Pointing vector, i.e., against the direction of light [17]. Thus, focusing the light at the point on the surface of the salt solution can create a local area with a more positive charge (potential). Since this method avoids the introduction of any impurities in the working solutions, this is a definite plus.

As a result, positively charged center was formed on the surface of the salt solution. After drying, this salt solution 
formed a picture of salt the rays diverge from such center (Figure 9) [1]. Thus, this assumption was partially confirmed.

In favor of this assumption is also evidenced by changes of the surfaces of the cubic or rhombic crystals occurring upon contact with water, which has a negative electric potential (Figure 10). Recall that cubic and rhombic crystals are formed in water with a positive potential (Figure 1).

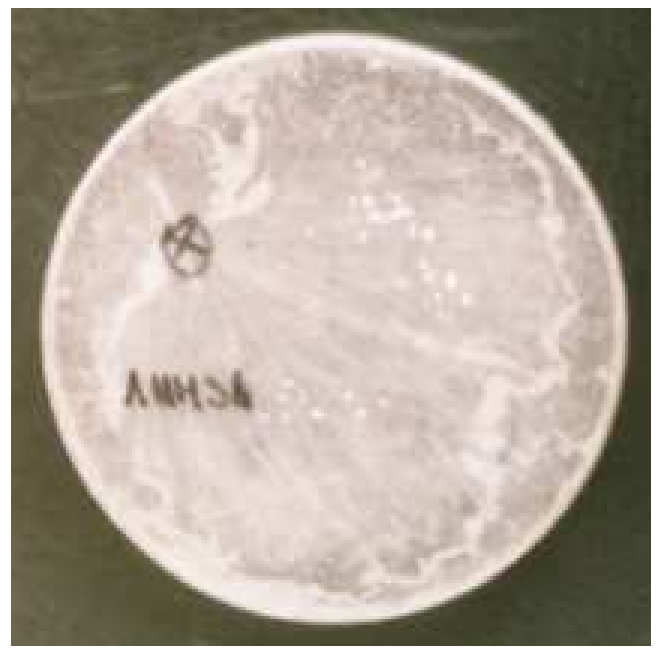

Figure 9. When the saline solution dries out, the place where the light was initially focused (marked with a cross) becomes the center of crystallization of needle-shaped crystals. [1].

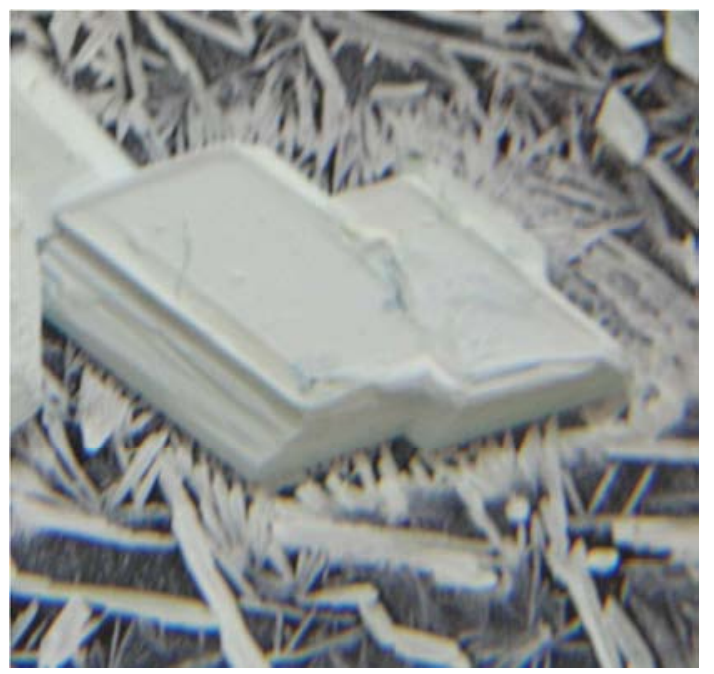

Figure 10. Upon contact with negatively charged water, on the surface of rhombic crystals of copper sulphate, whiskers are formed.

Considering the presented results, try to offer a reasonable mechanism for the formation of snowflakes in the clouds.

\subsection{A Possible Mechanism for the Formation of Snowflakes in the Clouds}

Considering the presented results, and especially the electric polarization of clouds (Figure 11) [18], it can be assumed that the formation of snowflakes in clouds occurs in two stages.

The first stage is the formation of small ice crystals in the upper part of the cloud, which has a positive charge (Figure
11). It should be noted that this assumption is fully consistent with known data about the place of the initial stage of the formation of snowflakes [19].

At the second stage clearly has to be a primary fall of snowflakes under the action of gravity. They will inevitably cross the lower edge of the cloud, which has a negative charge (Figure 11).

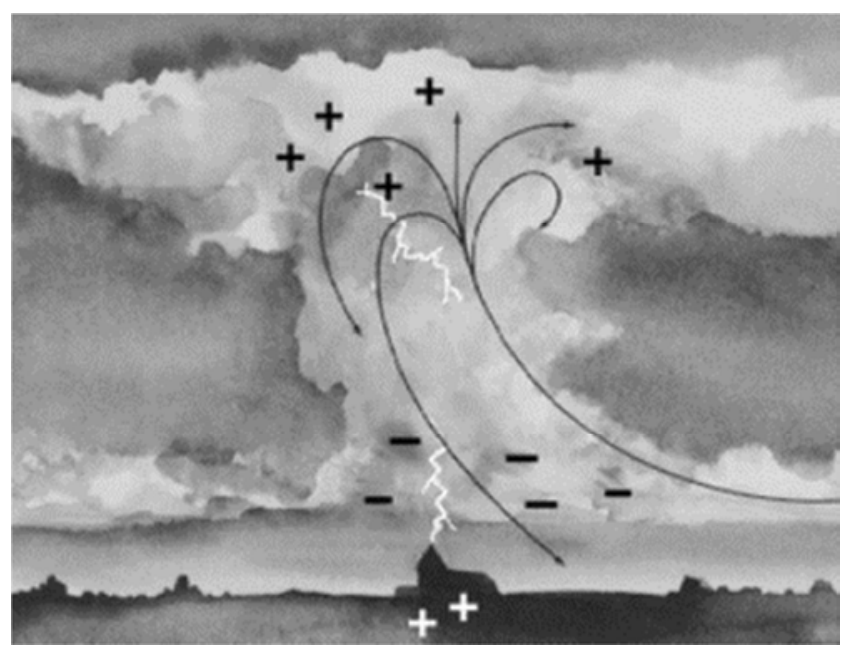

Figure 11. Polarization of clouds: the lower part of a typical cloud has a negative charge and the upper part has a positive charge [18].

Considering the presented results (Figures 8-10), it can be assumed that this completes the formation of snowflakes.

It is obvious that experimentally test the proposed mechanism of the formation of snowflakes is very difficult. For this reason, it was decided to simulate. This simulation consisted of two stages. In the first stage, crystals were obtained, formed after the evaporation of $\mathrm{NaCl}$ solution prepared with water with a positive potential. It is clear that these crystals had the shape close to cubic (Figure 12).

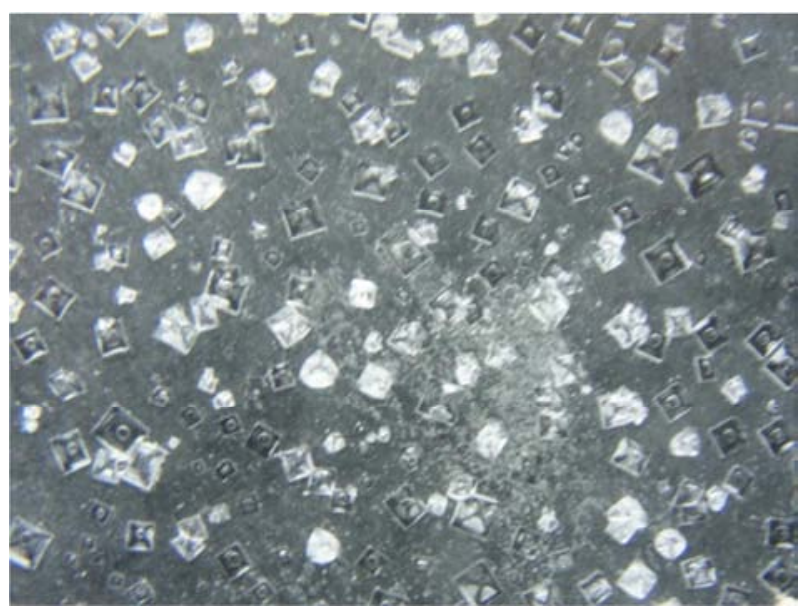

Figure 12. These are the cubic crystals formed after drying of the $\mathrm{NaCl}$ solution prepared with water with a positive potential.

In the second stage, the obtained cubic crystals of $\mathrm{NaCl}$ were poured in a volumetric cylinder filled with water with negative potential. The crystals that formed after the evaporation of the contents of this cylinder had obvious 
fragments of snowflakes (Figure 13).

Due to the results obtained, the proposed mechanism for the formation of snowflakes has a visual confirmation. One can hope that this is not just a visual coincidence. Accordingly, one can also hope that this visualization will provide a convincing answer to the old question about the origin of snowflakes [3-10].
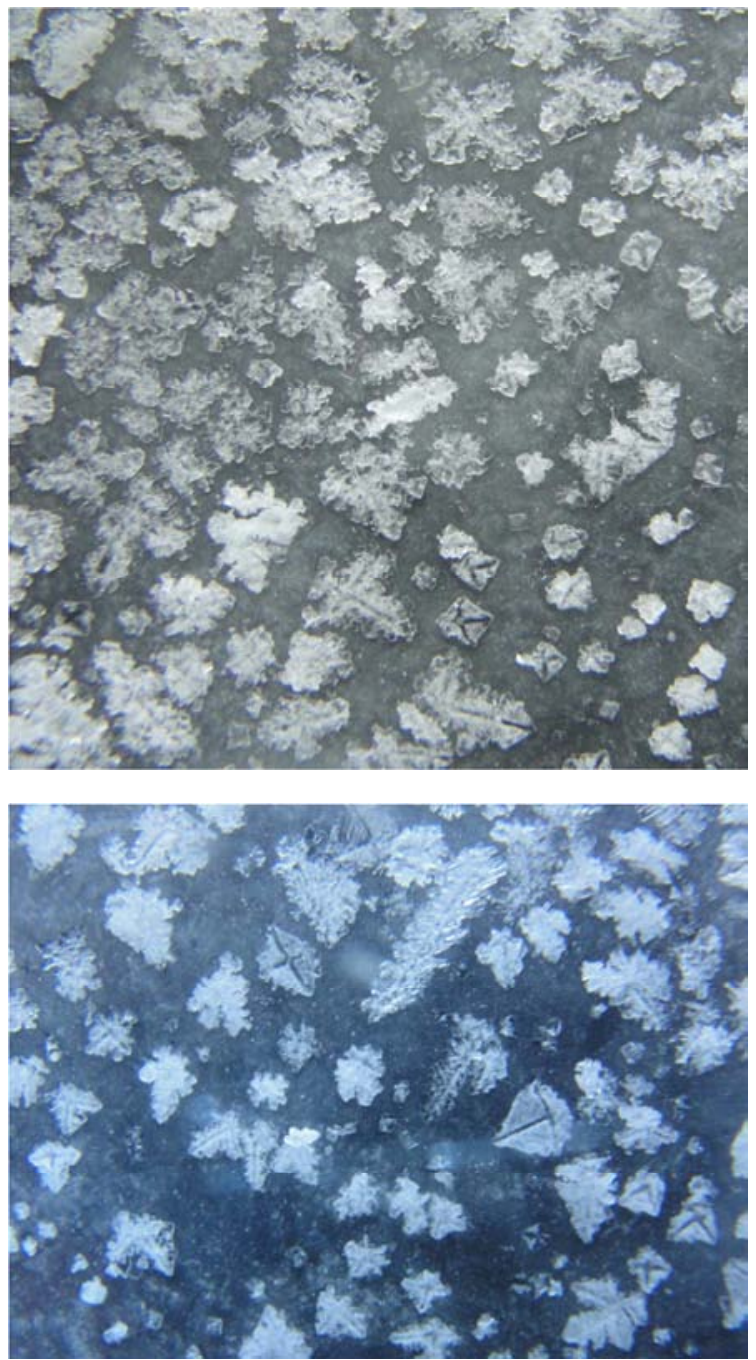

Figure 13. These are the cubic crystals of $\mathrm{NaCl}$ that have acquired fragments of snowflakes.

\section{Conclusion}

Forms of salt crystals are very sensitive to the electrical potential of the environment and can uniquely reflect this potential. Therefore, polymorphism of salt crystals can be used as an indicator of the electrical potential of the medium.

Thus, the knowledge of the described dependence makes it possible to explain some phenomena whose nature is still considered incomprehensible. For example, using this dependence, it is possible to explain the phenomena occurring in the storm glass, as well as the mechanism for the formation of snowflakes in the clouds.

\section{References}

[1] Pivovarenko Yu. V. (2016) Nature of the polymorphism of salt crystals in the aspect of arborization diagnostic method. Morphologia, 10 (1), 72-6.

[2] Pivovarenko Y. (2017) Potential-Dependent Changes of the Surface Tension of Water. Fluid Mechanics, 3 (4), 29-32.

[3] Warren I. P. (1863) Snowflakes: a chapter from the book of nature. Boston: American Tract Society.

[4] Nakaya U. (1954) Snow crystals: natural and artificial. Cambridge: Harvard University Press.

[5] Smalley I. J. (1963) Symmetry of Snow Crystals. Nature, 198 (4885), 1080-1081.

[6] Knight C. and Knight N. (1973) Snow crystals. Scientific American, 228 (1), 100-107.

[7] Petrenko V. F. and Whitworth R. W. (1999) Physics of Ice, Oxford University Press.

[8] Dovgaluk Yu. A. and Pershina T. A. (2005) Atlas of snowflakes (snow crystals). St Petersburg: Gidrometeoizdat (In Russian).

[9] Nelson J. (2008) Origin of diversity in falling snow. Atmos. Chem. Phys., 8, 5669-5682.

[10] Vijay P. Singh, Pratap Singh and Umesh K., Haritashya (2011) Encyclopedia of Snow; Ice and Glaciers. Dordrecht, Netherlands: Springer Science \& Business Media.

[11] Purcell E. (1971) Electricity and magnetism. BPC, 2. Moscow: Nauka. In Russian.

[12] Nekrasov B. V. (1974) Grounds general chemistry, 1, Moscow: Chemistry. In Russian.

[13] Fridrichsberg D. A. (1974) Course of colloid chemistry. Leningrad: Chemistry. In Russian.

[14] Lepeshinskaya, O. B. and Kosorotova M. V. (1946) Crystallization phenomena in living matter. Science and Life, 7, 7-11. In Russian.

[15] Mamaev O. (2015) Weather in a small flacon. Science and Life, 6, 81-86. In Russian.

[16] Kuznetsov V. V., Cherneva N. I. and Druzhin G. I. (2007) On the influence of cyclones on the atmospheric electric field of Kamchatka. Reports of the Academy of Sciences, 412 (4), 1-5.

[17] Crawford F. (1974) Waves. BCF, 3, Moscow: Nauka. In Russian.

[18] Feinman R., Leiton R., and Sands M. (1965) FLP, 5, Moscow: Mir. In Russian.

[19] Muchnik V. M. (1974) Physics of thunderstorms. St Petersburg: Gidrometeoizdat. In Russian. 